\title{
Erratum: Vol. 69, No. 5
}

In the report "Initial Public Health Response and Interim Clinical Guidance for the 2019 Novel Coronavirus Outbreak - United States, December 31, 2019-February 4, 2020," on page 142, the fourth sentence of the first paragraph under "Laboratory and Diagnostic Support" should have read "On February 4, 2020 the Food and Drug Administration issued an Emergency Use Authorization to enable emergency use of CDC’s 2019-nCoV Real-Time RT-PCR Diagnostic Panel."
On page 142, the first sentence of the first paragraph in the second column should have read "CDC is working closely with FDA and public health partners, including the Association of Public Health Laboratories, to rapidly share these tests domestically and internationally through CDC's International Reagent Resource (https://www.internationalreagentresource.org/)."

In addition, names of the members of the 2019-nCoV CDC Response Team were omitted. The names are included below.

\section{9-nCoV CDC Response Team}

Faruque Ahmed, National Center for Emerging and Zoonotic Infectious Diseases, CDC; Olivia Almendares, National Center for Immunization and Respiratory Diseases, CDC; Ashwin Belludi, National Center for Immunization and Respiratory Diseases, CDC; Isaac Benowitz, National Center for Emerging and Zoonotic Infectious Diseases, CDC; Chris Braden, National Center for Emerging and Zoonotic Infectious Diseases, CDC; Christina Carlson, Center for Global Health, CDC; Howard Chiou, Center for Surveillance, Epidemiology, and Laboratory Services, CDC; Nakia Clemmons, National Center for Immunization and Respiratory Diseases, CDC; Dave Daigle, Center for Global Health, CDC; Meghna Desai, Center for Global Health, CDC; Lindsey Duca, National Center for Chronic Disease Prevention and Health Promotion, CDC; Marc Fischer, National Center for Emerging and Zoonotic Infectious Diseases, CDC; Isaac Ghinai, Center for Surveillance, Epidemiology, and Laboratory Services, CDC; Carolyn Greene, National Center for Immunization and Respiratory Diseases, CDC; Cheri Grigg, National Center for Emerging and Zoonotic Infectious Diseases, CDC; Ardath Grills, National Center for Emerging and Zoonotic Infectious Diseases, CDC; Katherina Grusich, National Center for Immunization and Respiratory Diseases, CDC; Benjamin Hallowell, National Center for Immunization and Respiratory Diseases, CDC; Connor Hoff, National Center for Emerging and Zoonotic Infectious Diseases, CDC; Jesica Jacobs, Center for Surveillance, Epidemiology, and Laboratory Services, CDC; Bradley King, National Institute for Occupational Safety and Health, CDC; John MacArthur, Center for Global Health, CDC; Claire Mattison, National Center for Immunization and Respiratory Diseases, CDC; Jason McDonald, Center for Preparedness and Response, CDC; Tristan McPherson, Center for Surveillance, Epidemiology, and Laboratory Services, CDC; Alexander Millman, National Center for Immunization and Respiratory Diseases, CDC; Shannon Novosad, National Center for Emerging and Zoonotic Infectious Diseases, CDC; Mary Pomeroy, National Center for Emerging and Zoonotic Infectious Diseases, CDC; Noreen Qualls, National Center for Emerging and Zoonotic Infectious Diseases, CDC; Maryan Reynolds, National Center for Emerging and Zoonotic Infectious Diseases, CDC; Heather Rhodes, Center for Surveillance, Epidemiology, and Laboratory Services, CDC; Rajeev Sharma, Center for Global Health, CDC; Robert Simmonds, Center for Global Health, CDC; Rebekah Stewart, National Center for HIV/ AIDS, Viral Hepatitis, STD, and TB Prevention, CDC; Rebecca Sunenshine, Center for Preparedness and Response, CDC; Mark Tenforde, National Center for Immunization and Respiratory Diseases, CDC; Amra Uzicanin, National Center for Emerging and Zoonotic Infectious Diseases, CDC; Jennifer Verani, Center for Global Health, CDC; Florence Whitehill, National Center on Birth Defects and Developmental Disabilities, CDC; Kathryn Wilson, National Center for Emerging and Zoonotic Infectious Diseases, CDC; Jonathan Wortham, National Center for HIV/AIDS, Viral Hepatitis, STD, and TB Prevention, CDC. 